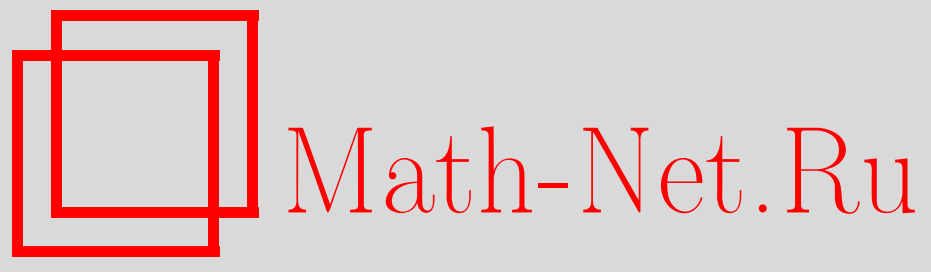

A. М. Чеботарев, А. Е. Теретенков, Операторные ОДУ и формула Фейнмана, Матем. заметки, 2012, том 92, выпуск 6, 943-948

DOI: https://doi.org/10.4213/mzm10151

Использование Общероссийского математического портала Math-Net.Ru подразумевает, что вы прочитали и согласны с пользовательским соглашением http://www . mathnet.ru/rus/agreement

Параметры загрузки:

IP : 52.205 .19 .152

26 апреля 2023 г., 17:31:19

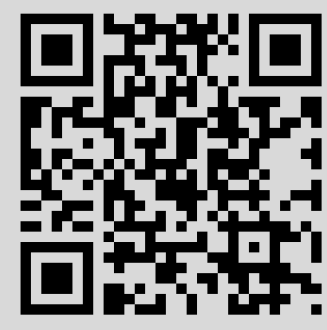




\section{Операторные ОДУ и формула Фейнмана}

\section{А. М. Чеботарев, А. Е. Теретенков}

Дифференцирование функций от некоммутирующих операторов, зависящих от параметра, по которому производится дифференцирование, усложняется, если операторы и их производные не коммутируют. Для вычисления производной $\dot{G}_{t}$ операторозначной экспоненты $e^{G_{t}}$ в случае $\left[G_{t}, \dot{G}_{t}\right] \neq 0$ Фейнман [1] использовал формулу, связывающую $G_{t}$ с левой производной $\dot{G}_{t}^{L}$ этого семейства $\left(\dot{G}_{t}^{L}: G_{t}^{L} e^{G_{t}}=(d /(d t)) e^{G_{t}}\right.$ (см. [2]), а также обсуждение в [3; с. 275 , формула (1.10)], и в [4]):

$$
\dot{G}_{t}^{L} \stackrel{\text { def }}{=}\left(\frac{d}{d t} e^{G_{t}}\right) e^{-G_{t}}=\lim _{\Delta t \rightarrow 0} \frac{1}{\Delta t} \int_{0}^{1} d s \frac{d}{d s} e^{s\left(G_{t}+\Delta t \dot{G}_{t}\right)} e^{-s G_{t}}=\int_{0}^{1} d s e^{s G_{t}} \dot{G}_{t} e^{-s G_{t}} .
$$

Аналогичный вид имеет правая производная

$$
\dot{G}_{t}^{R} \stackrel{\text { def }}{=} e^{-G_{t}}\left(\frac{d}{d t} e^{G_{t}}\right)=\int_{0}^{1} d s e^{-s G_{t}} \dot{G}_{t} e^{s G_{t}}=e^{-G_{t}} \dot{G}_{t}^{L} e^{G_{t}}=-\left(\frac{d}{d t} e^{-G_{t}}\right) e^{G t} .
$$

Последнее равенство вытекает из тождества $(d / d t) e^{-G_{t}} e^{G_{t}}=0$.

Например, если $G_{t}=\left(a^{\dagger}, C_{t} a\right)$ и $\left[C_{t}, \dot{C}_{t}\right] \neq 0$, где $C_{t}$ - семейство эрмитовых $(n \times n)$-матриц и $a^{\dagger}=\left\{a_{1}^{\dagger}, \ldots, a_{n}^{\dagger}\right\},\left\{a_{1}, \ldots, a_{n}\right\}$, то учитывая, что

$$
\begin{aligned}
& a_{t}^{\dagger}(s) \stackrel{\text { def }}{=} e^{s G_{t}} a^{\dagger} e^{-s G_{t}}=e^{s C_{t}^{T}} a^{\dagger}, \quad a_{t}(s) \stackrel{\text { def }}{=} e^{s G_{t}} a e^{-s G_{t}}=e^{-s C_{t}} a, \\
& e^{s G_{t}} \dot{G}_{t} e^{-s G_{t}}=e^{s\left(a^{\dagger}, C_{t} a\right)}\left(a^{\dagger}, \dot{C}_{t} a\right) e^{-s\left(a^{\dagger}, C_{t} a\right)}=\left(a_{t}^{\dagger}(s), \dot{C}_{t} a_{t}(s)\right)=\left(e^{s C_{t}^{T}} a^{\dagger}, \dot{C}_{t} e^{-s C_{t}} a\right),
\end{aligned}
$$

получаем

$$
\dot{G}_{t}^{L}=\int_{0}^{1} d s e^{s G_{t}} \dot{G}_{t} e^{-s G_{t}}=\left(a^{\dagger}, \int_{0}^{1} d s e^{s C_{t}} \dot{C}_{t} e^{-s C_{t}} a\right)=\left(a^{\dagger}, \dot{C}_{t}^{L} a\right) .
$$

Поэтому $\dot{G}_{t}^{L}=\left(a^{\dagger}, \dot{C}_{t}^{L} a\right)$ и

$$
\int_{0}^{1} d s e^{s C_{t}} \dot{C}_{t} e^{-s C_{t}}=\dot{C}_{t}^{L}
$$

Последнее соотношение можно рассматривать как неявное матричное ОДУ относительно $C_{t}$ с известной правой частью $\dot{C}_{t}^{L}$. Именно такая задача возникает далее при выводе многомодового варианта формулы Киржница-Боголюбова.

В общем случае из формулы (1) следует, что для вычисления $G_{t}$ нужно решить задачу Коши для неявной системы операторных ОДУ (ООДУ)

$$
\int_{0}^{1} d s e^{s G_{t}} \dot{G}_{t} e^{-s G_{t}}=\dot{G}_{t}^{L},\left.\quad G\right|_{t=0}=G_{0}
$$

Предполагается, что операторы $G_{t}^{L}$ известны. В частном случае, если операторы $G_{t}^{L}, \dot{G}_{t}^{L}$ и $G_{0}$ коммутируют, решением задачи (3) является функция $G_{t}=G_{t}^{L}$ и остается открытым лишь вопрос о его единственности.

Для построения численных методов решения операторных ОДУ порядка точности выше первого требуются формулы для производных высших порядков, которые могут быть получены путем дифференцирования и повторного применения формул (1), (3):

$$
\begin{aligned}
& \frac{d}{d t} e^{ \pm s G_{t}}= \pm \int_{0}^{s} d \sigma e^{ \pm \sigma G_{t}} \dot{G}_{t} e^{ \pm(s-\sigma) G_{t}}= \pm \int_{0}^{s} d \sigma e^{ \pm(s-\sigma) G_{t}} \dot{G}_{t} e^{ \pm s G_{t}}, \\
& \int_{0}^{1} d s e^{s G_{t}} \ddot{G}_{t} e^{-s G_{t}}=\ddot{G}_{t}^{L}+\int_{0}^{1} d s \int_{0}^{s} d \sigma\left[e^{s G_{t}} \dot{G}_{t} e^{-s G_{t}}, e^{\sigma G_{t}} \dot{G}_{t} e^{-\sigma G_{t}}\right] .
\end{aligned}
$$


Неявные уравнения для высших производных выводятся аналогично, а для получения явных формул необходима конструкция решений $X_{t}$ уравнений $\int_{0}^{1} d s e^{s G_{t}} X_{t} e^{-s G_{t}}=A_{t}$.

Для этой цели удобно использовать представление, в котором операторы $e^{ \pm s G_{t}}$ имеют наиболее простую форму. Если они эрмитовы или симметричны - их можно диагонализовать и в этом случае интегралы (1), (3), (4) вычисляются явно. Как будет показано ниже, единственность решения этих уравнений может нарушаться только при наличии у операторов $G_{t}$ точек чисто мнимого спектра, совпадающих по $\bmod 2 k \pi$.

В общем случае можно воспользоваться жордановым представлением операторов $G_{t}$ и соответствующим блочным представлением обкладок $e^{ \pm s G_{t}}$, которое также позволяет явным образом вычислить интегралы и свести задачу к решению системы линейных уравнений размера $\left(\operatorname{rank} G_{t}\right)^{2}$.

Обозначим через $J=J\left(\lambda_{1}, n_{1}, \ldots \lambda_{K}, n_{K}\right) \in \mathcal{M}(N)$ - жорданову матрицу, состоящую из $K$ клеток $J_{\alpha}$ ранга $n_{\alpha}, \sum_{\alpha} n_{\alpha}=N$. Известно, что $f\left(J_{\alpha}\right)$ вычисляется с помощью разложения $f$ в ряд Тейлора:

$$
\begin{aligned}
J_{\alpha} & =\left(\begin{array}{cccccc}
\lambda_{\alpha} & 1 & 0 & \ldots & 0 & 0 \\
0 & \lambda_{\alpha} & 1 & \ldots & 0 & 0 \\
\ldots & \ldots & \ldots & \ldots & \ldots & \ldots \\
0 & 0 & 0 & \ldots & \lambda_{\alpha} & 1 \\
0 & 0 & 0 & \ldots & 0 & \lambda_{\alpha}
\end{array}\right), \\
f\left(J_{\alpha}\right) & =\left(\begin{array}{cccc}
f\left(\lambda_{\alpha}\right) & \frac{1}{1 !} f^{(1)}\left(\lambda_{\alpha}\right) & \ldots & \frac{1}{\left(n_{\alpha}-1\right) !} f^{\left(n_{\alpha}-1\right)}\left(\lambda_{\alpha}\right) \\
0 & f\left(\lambda_{\alpha}\right) & \ldots & \frac{1}{\left(n_{\alpha}-2\right) !} f^{\left(n_{\alpha}-2\right)}\left(\lambda_{\alpha}\right) \\
\ldots & \ldots & \ldots & \ldots \\
0 & 0 & 0 & f\left(\lambda_{\alpha}\right)
\end{array}\right) .
\end{aligned}
$$

В частности, аналитическое выражение для экспоненты жордановой матрицы имеет вид

$$
\begin{gathered}
e^{s J}=\left(\begin{array}{cccc}
e^{s J_{1}} & 0 & \ldots & 0 \\
0 & e^{s J_{2}} & \cdots & 0 \\
\ldots & \cdots & \cdots & \cdots \\
0 & 0 & 0 & e^{s J_{K}}
\end{array}\right), \quad e^{s J_{\alpha}}=e^{s \lambda_{\alpha}} T_{\alpha}\left(s \lambda_{\alpha}\right), \\
T_{\alpha}(x)=\left(\begin{array}{cccc}
1 & \frac{x}{1 !} & \cdots & \frac{x^{k_{\alpha}-1}}{\left(k_{\alpha}-1\right) !} \\
0 & 1 & \cdots & \frac{x^{k_{\alpha}-2}}{\left(k_{\alpha}-2\right) !} \\
\cdots & \cdots & \cdots & \cdots \\
0 & 0 & 0 & 1
\end{array}\right)
\end{gathered}
$$

причем матричные элементы матрицы $T_{\alpha}(s)$ зависят от разности индексов и поэтому постоянны вдоль диагоналей.

Рассмотрим интеграл $X_{J} \stackrel{\text { def }}{=} \int_{0}^{1}\left(e^{s J} X e^{-s J}\right) d s$, в котором $(N \times N)$-матрица $X=\left\{x_{i j}\right\}$ разбита по строкам и столбцам на $K^{2}$ прямоугольных блоков $X_{\alpha \beta}$, соответствующих размерам жордановых клеток $T_{\alpha}$ и $T_{\beta}$, где

$$
X_{\alpha \beta}=\left\{x_{i j}\right\}, \quad N_{\alpha} \leqslant i \leqslant N_{\alpha}+n_{\alpha}, \quad N_{\beta} \leqslant j \leqslant N_{\beta}+n_{\beta} .
$$

Обратимое преобразование $\{a, j ; b, k\} \leftrightarrow\left\{a+(j-1) N_{\alpha}, k+(b-1) N_{\alpha}\right\}$ четверки индексов $a, b \in\{1, \ldots, K\}, k, j \in\{1, \ldots, N\}$ в пару $\left\{a+(j-1) N_{\alpha}, k+(b-1) N_{\alpha}\right\} \in\{1, \ldots, K N\}^{2}$, будем называть преобразованием $\mathfrak{D}_{42}=(D 4 \rightarrow D 2)$. Оно состоит из двух преобразований $\mathfrak{D}_{21}=(D 2 \leftrightarrow D 1):\{a, j\} \rightarrow m=a+(j-1) N_{\alpha}$. При этом $m \rightarrow\left\{a=m\left(\bmod N_{\alpha}\right)\right.$, $j=1+$ целая часть дроби $\left.m / N_{\alpha}\right\}$ является обратным преобразованием $\mathfrak{D}_{12}=(D 1 \rightarrow D 2)$. 
Нетрудно видеть, что структура жордановых обкладок обеспечивает отображение каждого блока $X_{\alpha \beta}$ в аналогичный блок матрицы $X_{J}$. Поэтому записывая матрицы $X$ и $X_{J}$ как векторы $\vec{X}=\mathfrak{D}_{21} X$ и $\overrightarrow{X_{J}}=\mathfrak{D}_{21} X_{J}$, компоненты которых собираются последовательно из строк каждого $n_{\alpha} \times n_{\beta}$ блока так, чтобы матричные элементы, входящие в один блок, следовали один за другим, получаем матричное блочно-диагональное представление четырехиндексного отображения $M_{J}: \vec{X} \rightarrow \overrightarrow{X_{J}}$ :

$$
M_{J} \stackrel{\text { def }}{=} \int_{0}^{1} M_{J}(s) d s, \quad M_{J}(s): \mathfrak{D}_{21}\left(e^{s J} X e^{-s J}\right)=\mathfrak{D}_{42}\left(e^{s J} \times e^{-s J^{T}}\right) \mathfrak{D}_{21} X \stackrel{\text { def }}{=} M_{J}(s) \vec{X} .
$$

Имеет место следующий замечательный факт.

Tеорема 1. 1. Имеем

$$
\operatorname{det} M_{J}(s)=1, \quad \operatorname{det} M_{J}=\prod_{\alpha, \beta=1}^{K}\left(\frac{e^{\lambda_{\alpha}-\lambda_{\beta}}-1}{\lambda_{\alpha}-\lambda_{\beta}}\right)^{n_{\alpha} n_{\beta}} .
$$

Более точно, матрицы $M_{J}(s)$ и $M_{J}$ - верхние треугольные с диагоналями, содержащими по $n_{\alpha} n_{\beta}$ элементов $\left\{e^{\left(\lambda_{\alpha}-\lambda_{\beta}\right) s}\right\}$ u $\left\{\left(e^{\lambda_{\alpha}-\lambda_{\beta}}-1\right) /\left(\lambda_{\alpha}-\lambda_{\beta}\right)\right\}$ соответственно.

2. Решение уравнения $\int_{0}^{1}\left(e^{s J} X e^{-s J}\right) d s=Y$ при условии

$$
\lambda_{\alpha}-\lambda_{\beta} \neq 0 \quad(\bmod 2 \pi i)
$$

сводится $\kappa$ обратимому преобразованию $\mathfrak{D}_{42}=(D 4 \rightarrow D 2)$ четырехиндексной матриць $e^{s J} \times e^{-s J^{T}}$ в двухиндексную невырожденную треуголъную матричу $M_{J}$, обращению $M_{J}$ u преобразованию $\mathfrak{D}_{21}=(D 1 \rightarrow D 2)$ вектора в матрииу

$$
X=\mathfrak{D}_{12}\left(M_{J}^{-1}\left(\mathfrak{D}_{21} Y\right)\right) .
$$

3. Если условие невырожденности (7) нарушено, то необходимым условием разрешимости уравнения $\int_{0}^{1}\left(e^{s J} X e^{-s J}\right) d s=Y$ является равенство нулю тех блоков $n_{\alpha} \times n_{\beta} u$ $n_{\beta} \times n_{\alpha}$ матрицы $Y$, для которых $\lambda_{\alpha}-\lambda_{\beta}=0(\bmod 2 \pi i)$. В этом случае соответствующие блоки решения $X$ могут быть выбраны произвольным образом.

ДоказАтельство. Справедливость утверждения теоремы о треугольном виде $M_{J}$ была замечена в процессе аналитических вычислений на компьютере. Рассмотрим аналитическое доказательство теоремы.

Поскольку переход от четырехиндексного отображения к обычному матричному представлению блоки $X_{\alpha \beta}$ отображаются в блоки $\left(X_{J}\right)_{\alpha \beta}$ такого же размера, достаточно рассмотреть преобразование произвольного блока.

Из определения жордановых блоков имеем следующие равенства:

$$
\begin{gathered}
\int_{0}^{1}\left(e^{s J_{\alpha}} X_{\alpha \beta} e^{-s J_{\beta}}\right)_{k j} d s=\sum_{a=k}^{N_{\alpha}+n_{\alpha}} \sum_{b=N_{\beta}}^{j} \int_{0}^{1} d s e^{\left(\lambda_{\alpha}-\lambda_{\beta}\right) s} \Lambda_{k j}^{a b}(s) x_{a b}, \\
\Lambda_{k j}^{a b}(s) \stackrel{\text { def }}{=} \begin{cases}(-1)^{j-b} \frac{s^{a-k}}{(a-k) !} \frac{s^{j-b}}{(j-b) !}, & N_{\alpha} \leqslant k \leqslant a \leqslant N_{\alpha}+n_{\alpha}, N_{\beta} \leqslant b \leqslant j \leqslant N_{\beta}+n_{\beta}, \\
0 & \text { в остальных случаях. }\end{cases}
\end{gathered}
$$

С помощью замены индексов $\{a, j ; b, k\} \leftrightarrow\left\{a+(j-1) N_{\alpha}, k+(b-1) N_{\alpha}\right\}$ преобразуем четырехиндексную матрицу $\Lambda_{k j}^{a b}(s)$ в верхнюю треугольную:

$$
M_{k+(b-1) N_{\beta}, a+(j-1) N_{\beta}}(s) \stackrel{\text { def }}{=} \mathfrak{D}_{42} e^{\left(\lambda_{\alpha}-\lambda_{\beta}\right) s} \Lambda_{k j}^{a b}(s) .
$$

Из $(10)$ следует, что в области $a+(j-1) N_{\beta}<k+(b-1) N_{\beta}$ матрица $M_{k+(b-1) N_{\beta}, a+(j-1) N_{\beta}}(s)$ равна нулю и $e^{\left(\lambda_{\alpha}-\lambda_{\beta}\right) s}$ на диагонали. Поэтому произведение диагональных элементов равно $e^{\sum_{\alpha, \beta=1}^{K}\left(\lambda_{\alpha}-\lambda_{\beta}\right) s}=1$. 
Для получения $M_{J}$ нужно умножить каждый блок на интеграл по $s$ от $e^{\left(\lambda_{\alpha}-\lambda_{\beta}\right) s} \Lambda_{k j}^{a b}(s)$, который равен $\left(e^{\lambda_{\alpha}-\lambda_{\beta}}-1\right) /\left(\lambda_{\alpha}-\lambda_{\beta}\right)$ и встречается $n_{\alpha} n_{\beta}$ раз на диагонали.

Если выполнены условия второго утверждения теоремы, то треугольная матрица $M_{J}$ обратима. Если $\lambda_{\alpha}-\lambda_{\beta}=0(\bmod 2 \pi i)$ и соответствующие блоки матрицы $\left(X_{J}\right)_{\alpha \beta}$ (правой части уравнения) должны также быть равны нулю, а элементы блока решения $X_{\alpha, \beta}$ можно задать произвольно.

Если семейство $G_{t}$ имеет спектральные разложения, типичные для эрмитовых либо симметричных операторов, то в этом простейшем случае

$$
G_{t}=\sum_{n} \lambda_{n}(t) \begin{cases}\left|\xi_{n}(t)\right\rangle\left\langle\xi_{n}(t)\right|, & \left\langle\xi_{j}, \xi_{k}\right\rangle=\delta_{j k}, \\ \left.\mid \xi_{n}(t)\right)\left(\xi_{n}(t) \mid,\right. & \left(\xi_{j}, \xi_{k}\right)=\delta_{j k},\end{cases}
$$

где $\lambda_{n}(t) \in \mathbb{C},\left\langle\xi_{j}, \xi_{k}\right\rangle$ - полуторалинейное скалярное произведение, либо $\left(\xi_{j}, \xi_{k}\right)$ - билинейная квадратичная форма (являющаяся скалярным произведением на множестве векторов с вещественными компонентами), а $\left\{\xi_{n}(t)\right\}$ - система векторов ортонормированная относительно соответствующей полуторалинейной либо квадратичной формы, то система (3) может быть разрешена относительно $\dot{G}_{t}$ и записана в виде системы операторных ОДУ, которую можно стандартными методами диагонализовать численно и в некоторых частных случаях - аналитически.

Предположим, что матрица $G$ допускает диагонализацию с помощью обратимого преобразования $U: G \rightarrow G_{U}=U G U^{-1}=\left\{\lambda_{k} \delta_{k j}\right\}_{1 \leqslant j, k \leqslant K}$ и рассмотрим сравнительно простую конструкцию решения уравнения относительно матрицы $X$ :

$$
\int_{0}^{1} d s e^{s G} X e^{-s G}=F
$$

Теорема 2. 1) Если оператор $X$ является решением уравнения (12), то он также удовлетворяет уравнению

$$
e^{G} X e^{-G}-X=[G, F] .
$$

Если $X$ - решение уравнения (12), то $X+\phi$ также является решением (13) с любой матрищей $\phi$, коммутирующей $c G$.

2) Если оператор $G$ допускает разложение (11) и матрица $\Lambda$,

$$
\Lambda_{j k}=\int_{0}^{1} d s e^{s\left(\lambda_{j}-\lambda_{k}\right)}=\frac{e^{\lambda_{j}-\lambda_{k}}-1}{\lambda_{j}-\lambda_{k}},
$$

не имеет нулевых элементов, то единственное решение уравнения (12) имеет вид

$$
X=U^{-1}\left(V \circ F_{U}\right) U, \quad V_{j k}=\Lambda_{j k}^{-1}, \quad F_{U} \stackrel{\text { def }}{=} U F U^{-1},
$$

где $A \circ B$ - поэлементное произведение матрич (произведение Адамара), не зависящее от порядка сомножителей, сохраняющее эрмитовость, симметричность и положительную определенность сомножителей.

3) Если выполнены условия n.2, то уравнения (12) и (13) имеют общее решение (15), которое выделено условием равенства диагоналей матрии, $X_{U}=U X U^{-1}$ и $F_{U}$.

Доказательство. 1) Пусть $X$ - решение (12). Из (12) следует уравнение (13):

$$
\begin{aligned}
e^{G} X e^{-G}-X & =\int_{0}^{1} d s \frac{d}{d s}\left(e^{s G} X e^{-s G}\right) \\
& =G \int_{0}^{1} d s e^{s G} X e^{-s G}-\int_{0}^{1} d s e^{s G} X e^{-s G} G=G F-F G,
\end{aligned}
$$

обе части которого на меняются при сдвигах $X$ на матрицу, коммутирующую с $G$. 
2) Для дальнейшего анализа задачи удобно перейти к представлению $X \rightarrow X_{U}=$ $U X U^{-1}$, в котором диагонализуются операторы $e^{ \pm s G}$ :

$$
U F U^{-1}=F_{U}=\int_{0}^{1} d s e^{s \lambda} X_{U} e^{-s \lambda}=X_{U} \circ \int_{0}^{1} d s E(s), \quad E_{j k}(s) \stackrel{\text { def }}{=} e^{s\left(\lambda_{j}-\lambda_{k}\right)},
$$

где $e^{s \lambda}$ - диагональная матрица с диагональными элементами $e^{s \lambda_{k}}$ и $E(s)$ - матрица ранга 1 с элементами $E_{j k}(s)$. Зависимость от $s$ каждого элемента матрицы $E(s)$ явно задана, поэтому после интегрирования с учетом (14) получаем равенство $\left(F_{U}\right)_{j k}=\left(X_{U}\right)_{j k} \Lambda_{j k}$. Отсюда следует (15).

Решение (15) можно получить путем диагонализации тождества

$$
U F U^{-1}=F_{U}=\int_{0}^{1} d s e^{s \lambda} X_{U} e^{-s \lambda}=X_{U} \circ \int_{0}^{1} d s E(s), \quad E_{j k}(s) \stackrel{\text { def }}{=} e^{s\left(\lambda_{j}-\lambda_{k}\right)},
$$

Поскольку $E_{j j}(s)=\Lambda_{j j}=V_{j j}=1$, диагональные элементы $F_{U}$ и $X_{U}$ совпадают.

Доказательство сходных утверждений можно найти в [4], где также использовалось коммутативное поэлементное произведение Адамара $A \circ B$ матриц $A$ и $B$.

$3)$ В представлении, диагонализующем $G$, уравнение (13) имеет вид

$$
\left(X_{U}\right)_{j k}\left(e^{\lambda_{j}-\lambda_{k}}-1\right)=\left(F_{U}\right)_{j k}\left(\lambda_{j}-\lambda_{k}\right),
$$

причем сдвиг $X_{U}$ на любую диагональную матрицу не нарушает равенство. В этом же представлении обращаем обкладки $U, U^{-1}$ и получаем требуемый результат:

$$
\left(X_{U}\right)_{j k}=\left(F_{U}\right)_{j k} \frac{\lambda_{j}-\lambda_{k}}{e^{\lambda_{j}-\lambda_{k}}-1}=\left(F_{U}\right)_{j k} V_{j k}, \quad X=U^{-1}\left(V \circ F_{U}\right) U .
$$

который совпадает с $(15)$ и обеспечивает равенство диагоналей $F_{U}$ и $X_{U}$. Таким образом, если выполнены условия п. 2, то уравнение (12) эквивалентно задаче (13) с "краевым" условием $\operatorname{diag}(F-X)_{U}=0$.

С точки зрения численной реализации, формула (15) или уравнение (13) с "краевым" условием для $X$ проще уравнения (3), так как они не требует вычисления операторозначных интегралов и решения нелинейных ОДУ неразрешенных относительно производной и позволяют записать решение задачи (3) в виде

$$
\begin{gathered}
G_{t}=G_{0}+\int_{0}^{t} d \tau U^{-1}(\tau)\left(V(\tau) \circ F_{U}(\tau)\right) U(\tau), \\
\Lambda(\tau)=U(\tau) \frac{e^{\lambda_{j}(\tau)-\lambda_{k}(\tau)}-1}{\lambda_{j}(\tau)-\lambda_{k}(\tau)} U^{-1}(\tau), \quad F_{U}(\tau)=U(\tau) F(\tau) U^{-1}(\tau), \quad V_{j k}(\tau)=\Lambda_{j k}^{-1}(\tau),
\end{gathered}
$$

где $\Lambda(\tau)$ - диагональная матрица, а $U(\tau)$ - соответствующее диагонализующее преобразование, а $V$ - матрица, состоящая из элементов $\Lambda_{k j}^{-1}$.

Матрицы $\Lambda$ и $V$ в (15) корректно определены при любых значениях $\left\{\lambda_{k}\right\}$ в случае вещественного спектра. Однако, если нарушается предположение $\Lambda_{j k} \neq 0$, т.е. если

$$
\mathcal{K} \stackrel{\text { def }}{=} \bigcup_{(j, k)}\left\{(j, k): \lambda_{j} \neq \lambda_{k}, \lambda_{j}-\lambda_{k}=0(\bmod 2 \pi i)\right\} \neq \varnothing,
$$

где $\left\{\lambda_{j}\right\}$ - спектр оператора $G_{U}$, то необходимое условие разрешимости уравнения

$$
\int_{0}^{1} d s e^{s G} X e^{-s G}=F
$$

состоит в том, что $\left.F_{U}\right|_{\mathcal{K}}=0$. При этом значения $X_{U}$ в точках $\mathcal{K}$ могут быть заданы произвольным образом, так что их выбор имеет смысл задания граничного условия. Ограничения на $\left.F_{U}\right|_{\mathcal{K}}$ зависят как от $F$, так и от $G$ и поэтому могут характеризовать либо 
класс корректности правых частей $F$, либо допустимое множество матриц $G$, играющих роль начального условия, определяющего последующие значения пар $\left\{\dot{G}_{t}, G_{t+\Delta t}\right\}$. Оба условия легко сформулировать в исходном базисе в терминах билинейных форм матриц $F$ и $G$.

Будем говорить, что задача (17) имеет тривиалъную границу, если $\mathcal{K}=\varnothing$, и что она корректна, если $\left.F_{U}\right|_{\mathcal{K}}=0$. Если граница нетривиальна, но задача поставлена корректно, то ее минимальным решением будем называть матрицу $X^{\text {min }}$, удовлетворяющую уравнению (17) и граничному условию $\left.X_{U}\right|_{\mathcal{K}}=0$.

Теорема 3. Общее решение задачи (17) можно представить в виде

$$
X=X^{\mathrm{min}}+\sum_{(j, k) \in \mathcal{K}} c_{j k} U^{-1} E_{j k} U,
$$

где $c_{j k}$ - произвольные константы, а $E_{j k}$ - матрица с единственным ненулевым элементом в $k$-м столбие и $j$-й строке, равным 1.

Доказательство. Нетрудно видеть, что $\int_{0}^{1} d s e^{s G} X^{\min } e^{-s G}=F$ в силу (15). Заметим, что

$$
\Lambda_{j k} E_{j k}=\frac{e^{\lambda_{j}-\lambda_{k}}-1}{\lambda_{j}-\lambda_{k}} E_{j k} \equiv 0
$$

для любых $j, k$ потому, что либо $E_{j k}=0$, либо $e^{\lambda_{j}-\lambda_{k}}=1$ при $\lambda_{j} \neq \lambda_{k}$. Следовательно, действие операторных обкладок $U, U^{-1}$ не изменяет правую часть этого равенства. Поэтому $\int_{0}^{1} e^{s \lambda} E_{j k} e^{-s \lambda} d s \equiv 0$ и

$$
0 \equiv \int_{0}^{1} U e^{s \lambda} E_{j k} e^{-s \lambda} U^{-1} d s=\sum_{j, k} \int_{0}^{1} e^{s G} U E_{j k} U^{-1} e^{-s G} d s=\int_{0}^{1} e^{s G}\left(X-X^{\mathrm{min}}\right) e^{-s G} d s .
$$

\section{СПИСОК ЦИТИРОВАННОЙ ЛИТЕРАТУРЫ}

[1] R. P. Feynman, Phys. Rev. (2), 84:1 (1951), 108-128. [2] R. M. Wilcox, J. Math. Phys., 8:4 (1967), 962-982. [3] М. В. Карасев, В. П. Маслов, Нелинейные скобки Пуассона. Геометрия и квантование, Наука, M., 1991. [4] I. Najfeld, T. F. Havel, Adv. Appl. Math., 16:3 (1995), 321-375.

А. М. Чеботарев

Московский государственный университет им. М. В. Ломоносова

E-mail: chebotarev@phys.msu.ru

\section{А. Е. Теретенков}

Московский государственный университет им. М. В. Ломоносова

E-mail: taemsu@mail.ru
Поступило

24.05.2012 